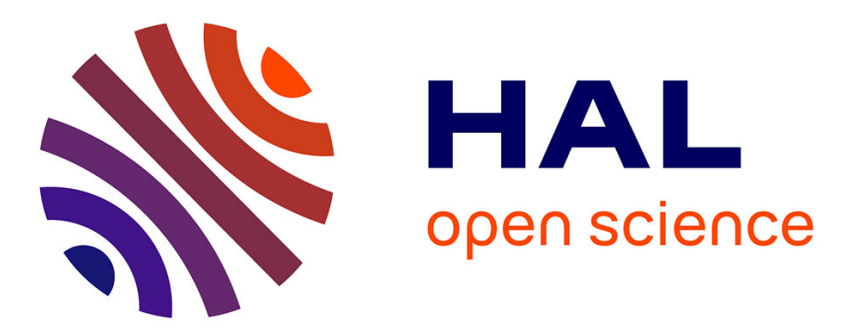

\title{
Stable and Reactive Centering in Conduits for Karstic Exploration
}

Adrien Lasbouygues, Lionel Lapierre, David Andreu, Josue Lopez Hermoso, Hervé Jourde, Benoît Ropars

\section{To cite this version:}

Adrien Lasbouygues, Lionel Lapierre, David Andreu, Josue Lopez Hermoso, Hervé Jourde, et al.. Stable and Reactive Centering in Conduits for Karstic Exploration. ECC 2014 - 13th European Control Conference, Jun 2014, Strasbourg, France. pp.2986-2991, 10.1109/ECC.2014.6862278 lirmm01071307

\section{HAL Id: lirmm-01071307 https://hal-lirmm.ccsd.cnrs.fr/lirmm-01071307}

Submitted on 3 Oct 2014

HAL is a multi-disciplinary open access archive for the deposit and dissemination of scientific research documents, whether they are published or not. The documents may come from teaching and research institutions in France or abroad, or from public or private research centers.
L'archive ouverte pluridisciplinaire HAL, est destinée au dépôt et à la diffusion de documents scientifiques de niveau recherche, publiés ou non, émanant des établissements d'enseignement et de recherche français ou étrangers, des laboratoires publics ou privés. 


\section{Stable and reactive centering in conduits for karstic exploration}

\author{
Adrien Lasbouygues, \\ Lionel Lapierre, David Andreu \\ and Josue Lopez Hermoso \\ LIRMM, \\ University Montpellier II \\ Montpellier, France \\ Email: adrien.lasbouygues@lirmm.fr
}

\author{
Herve Jourde \\ HydroSciences Montpellier, \\ University Montpellier II \\ Montpellier, France \\ Email: herve.jourde@univ-montp2.fr
}

\author{
Benoit Ropars \\ Ciscrea \\ Toulon, France \\ Email: bro@ciscrea.fr
}

\begin{abstract}
Karst groundwaters provide fresh water resources to supply cities. In the case of deep groundwater resources around the Mediterranean basin there is a need to explore karst aquifers and gather data about their structure. Robots are well adapted for this task especially when exploring karst conduits at depths greater than $200 \mathrm{~m}$. However, as karst aquifers environment is very complex, robots need to exhibit a collection of abilities to ensure a safe exploration. Among them, being able to "center" safely within the karst conduits and avoid collisions is a key point to ensure the robot safety during its mission. We propose a new reactive way to do so, based on the Deformable Virtual Zone. This allows us to model the interaction of the robot with its environment as a physics inspired equation. We also prove the stability of this controller.
\end{abstract}

\section{INTRODUCTION}

Karst aquifers are one of the most important sources of groundwater in the world. Thus they could provide an access to fresh water in regions, such as the karstic Mediterranean basin, where water is a scarce resource. However, these groundwaters are currently widely underexploited [1]. Indeed hydrogeologists need to know quite precisely the geomorphology of the karst system in order to optimize water resource management and, for instance, avoid the depletion of a karst spring because of an uncontrolled pumping. However, because of their complex structure, karst aquifers exhibit particular hydrogeological behaviour; more information about this structure can thus help to get a better understanding of groundwater dynamics [2].

We will focus on the issue of mapping main conduits of the drainage network of karst aquifers. It can help to find for instance the best place to drill a borehole in order to pump with the best efficiency the groundwater within the aquifer. Geophysical methods, such as georadar, have a limited range and thus can only provide limited information on the conduit network. Another commonly used mapping method is electromagnetic positioning where a diver puts a solenoid or a magnet in the conduit [2]. But this method is limited due to the restrictions enforced on the use of divers in karst aquifers because of many deadly accidents.

Thus Autonomous Underwater Vehicles (AUVs) become a solution for exploring the phreatic (i.e. filled with water) zones of karst aquifers. However, they shall be equipped with both sensors and control algorithms affording them the capacity to explore safely the karst conduits. We are here interested in centering the robot in such a karst conduit thus avoiding the robot collision with the walls of the conduit.

In [3] and [4], robots for pipe inspection are presented. They mostly exhibit locomotion systems such as wheels or legs that can be used thanks to the regular shape of the pipes. These systems allow the robots to explore safely the pipes but the complexity and irregularity of karstic conduits make these solutions unpractical. Controls proposed in [5] or [6] for corridor navigation could also be extended to $3 \mathrm{D}$ environments. But these solutions are developed for structured environments. Thus they are not adapted to the high complexity of karstic environments.

Solutions proposed for underwater cave exploration, such as the one presented in [7] and [8], are also limited because they generally assume that the robot is not surrounded by close walls. They are based on wall following and thus provide no sufficient guaranty for centering when the robot is closely surrounded by walls.

This paper presents a new control algorithm allowing to reactively reach a secure point in the conduit and based on the Deformable Virtual Zone (DVZ) principle and the representation of the robot-environment interaction by a physics inspired second order mass-spring-damper system. In the sequel this point will be called "center" of the karst conduit (even if it's not a real center because the karst environments are rarely cylindrical). In additon to the control algorithm, we present a proof of stability and a reflexion on how to tune the control algorithm parameters.

This paper is organized as follows. In section II, we introduce the problem statement and present the hypotheses made. In section III, the DVZ principle is detailled and a study on its parameters' value is made. The proposed control approach and its stability are presented in section IV and simulation results in section V. Finally this paper ends with a conclusion and an opening towards future work.

\section{PROBLEM STATEMENT AND HYPOTHESES}

\section{A. The AUV and its sensors}

Let us consider the AUV presented in Figure 1 which has to navigate through a karst conduit. It is based on the Jack 


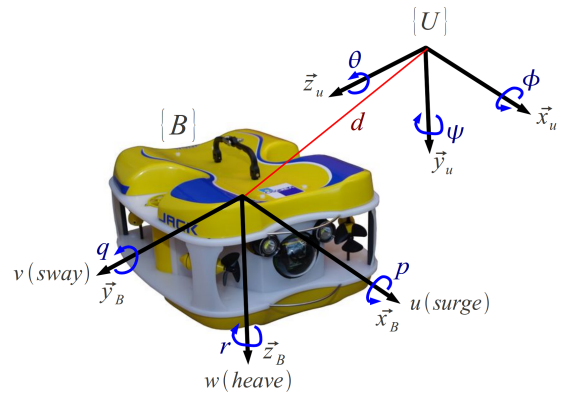

Fig. 1. Jack system

system manufactured by the Ciscrea company.

Let $\{B\}:=\left\{\overrightarrow{x_{B}}, \overrightarrow{y_{B}}, \overrightarrow{z_{B}}\right\}$ be its body axis frame and $\{U\}:=\left\{\overrightarrow{x_{U}}, \overrightarrow{y_{U}}, \overrightarrow{z_{U}}\right\}$ a global coordinate frame.

$\mathbf{d}=[x, y, z]$ is the position of $\{B\}$ with respect to $\{U\}$.

$[u, v, w]$ defines the linear system velocities in $\{B\}$. $[p, q, r]$ denotes the angular velocities around each of the axis of $\{B\} .[\phi, \theta, \psi]$ defines the vehicle attitude, respectively, the roll, pitch and yaw angles.

The AUV is equipped with a proximity sensor (here a profiling sonar) of sampling period $T_{c}$, with a range $d_{\text {max }_{\text {capt }}}$, a minimal detection distance $d_{\text {min }_{\text {capt }}}$ and $N_{R}$ rays. A measurement, which is the discretization of the current karst section, is given by :

$$
M_{d}=\left\{\left(d_{i}, \alpha_{i}\right), i=1, . ., N_{R}\right\}
$$

where $\alpha_{i}$ is the angle of the ray $i$ with respect to the $\overrightarrow{y_{B}}$ axis and the measured distance is such that :

$$
d_{\text {min }_{\text {capt }}} \leq d_{i} \leq d_{\text {max }_{\text {capt }}}
$$

The controller must also be able to know $v, w$ and the value of $\phi$ by using the appropriate sensor (Inertial Measurement Unit integration or Doppler Velocity Log).

\section{B. From a $3 D$ to a $2 D$ problem}

Assumption $1: u$ is controlled independently to set the horizontal distance between two sonar measurements, $\Delta_{\text {mes }}$, given by :

$$
\Delta_{m e s}=u * T_{c}
$$

$\Delta_{m e s}$ should be both compliant to any mission objective and small enough to ensure the environment will not change too much between two consecutive measurements. Moreover, by using a dedicated sensor, the control of $u$ has to ensure that the robot is stopped if the conduit is either too small for the robot to go through or a dead end.

The control of the two remaining $\operatorname{DoF} \theta$ and $\psi$ is designed to ensure that the direction of the conduit coincides with $\overrightarrow{x_{B}}$ and that $r$ and $q$ reach 0 .

Assumption 2 : Thus we limit our study to the $\left\{\overrightarrow{y_{B}}, \overrightarrow{z_{B}}\right\}$ plane bringing the problem back to a $2 \mathrm{D}$ problem, as shown in Figure 2, with the karst profile changing through time.

Moreover, as the interaction between the controler presented in Assumption 1 and the DVZ-based centering controller is beyond the scope of this paper, we will assume that the other controller has reached its steady state and thus

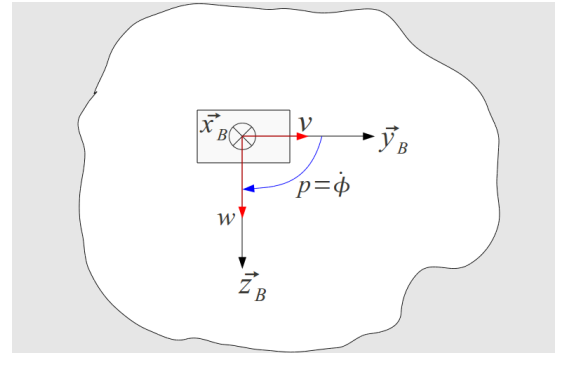

Fig. 2. The 2D problem statement

$r=0$ and $q=0$. So we can write the kinematic relation between $\dot{\phi}$ and $p$ as :

$$
\dot{\phi}=p
$$

Assumption 3 : The simplified inverse dynamic model, where the coupling terms are neglected, is :

$$
\begin{gathered}
F_{v}=m_{v} \dot{v}-d_{v} v \\
F_{w}=m_{w} \dot{w}-d_{w} w \\
\Gamma_{p}=m_{p} \dot{p}-d_{p} p
\end{gathered}
$$

where $m_{v}, m_{w}$ and $m_{p}$ are the total masses (dry mass + added mass or inertia) along each motion axis. $d_{v}, d_{w}$ and $d_{p}$ are the quadratic damping terms for each motion axis. We assume that this model is exact (i.e. its parameters are precisely known).

Under these assumptions, the control has two objectives : ensure that the robot reaches the "center" of the conduit and set its roll, $\phi$, to a desired value $\phi^{d}$.

\section{Assumptions on the Karst}

Finally we have to make a number of assumptions on the karst conduit in order to ensure that the robot can indeed reach the "center" of the conduit.

Assumption 4 : The conduit is filled with water. Thus the AUV can reach the karst "center". However, uncertainties related to water currents are neglected in this paper.

Moreover, to be sure that the robot can find this "center", it must be able to view the entire conduit outline.

Assumption 5 : The minimal, $d_{\text {min } n_{k a r s t}}$, and maximal,

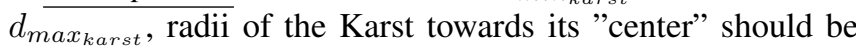
such that :

$$
d_{\text {min }_{\text {capt }}}<d_{\text {min }_{\text {karst }}}<2 * d_{\text {max }_{\text {karst }}}<d_{\text {max }_{\text {capt }}}
$$

Assumption 6: The robot must always be in the set of points such that the minimal distance of the robot to any walls $d_{\text {min }_{\text {walls }}}$ respects the condition :

$$
d_{\text {min }_{\text {capt }}} \leq d_{\text {min }_{\text {walls }}}
$$

Assumption 7 : Sometimes the karst conduit shape may be very concave (see Figure 3). If there is enough space for the robot to get through a part of the conduit, the sensor will not detect the parts of the conduit that lie beyond the concavity. Thus, from the robot perception, the conduit will be brought back to a shape respecting the previous assumptions.

Thus conduits such as the one presented in Figure 3 will not be considered in simulation and theory. 


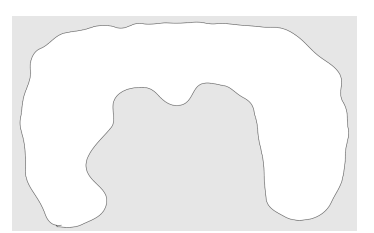

Fig. 3. A very concave conduit profile

\section{THE DVZ AND ITS PARAMETERS}

A. The DVZ : a representation of the interaction between the robot and its environment

Presented in [9] for obstacle avoidance, the DVZ (Deformable Virtual Zone) is a representation of the interaction between the robot and its environment. The DVZ is a zone, with a chosen geometric shape, surrounding the robot and which moves according to the robot dynamics. The intrusion of an obstacle deforms the DVZ shape. Then the DVZ reacts to this intrusion according to its own virtual dynamics in order to maintain its original shape. The robot control is finally computed to follow the DVZ required movement.

Thus we need to choose the DVZ shape and dynamics. Our DVZ will be a circle of radius $R_{D V Z}$. The DVZ dynamics is chosen so that the control along the robot's motions axes will behave like a mass-spring-damper system. Its well-known equation is :

$$
m \ddot{a}=-K a-c \dot{a}
$$

where $a$ represents the displacement of $m$ along the $\overrightarrow{a_{S}}$ axis.

The idea of using a force generated by a spring-like system was also proposed in [10] where impedance control uses a force generated by the robot-obstacle distance to compute the control required to avoid obstacles. However, thanks to the DVZ approach, we can use explicitly the mass-springdamper equation in the control algorithm. This allows us to study in an easier way our system parameters, since they have a physical related meaning and are not abstract coefficients. The DVZ approach allows us a simpler management of the control when the robot is surrounded by obstacles.

\section{B. Modifications to the DVZ}

In previous part, we explained that the DVZ reacts to shape deformations by trying to recover its original shape. However, when the DVZ is surrounded by obstacles it cannot recover its shape. This is what happens in the karst. Instead, the DVZ controller will drive the system to reach the point where all the intrusions compensate each other. This point will be such that the force resulting from all the intrusions (see Figure 6 ), $\vec{F}_{R}$, is null.

\section{Tuning of the DVZ radius}

As presented previously, our DVZ behaviour is a circle of radius $R_{D V Z}$. The intrusion-generated force, shall not depend on the value of $R_{D V Z}$ but only on the distance of the robot to the conduit "center". Figure 4 shows the relationship between $R_{D V Z}$ and the intrusion-generated force when the robot is close to the conduit "center" as shown in the left

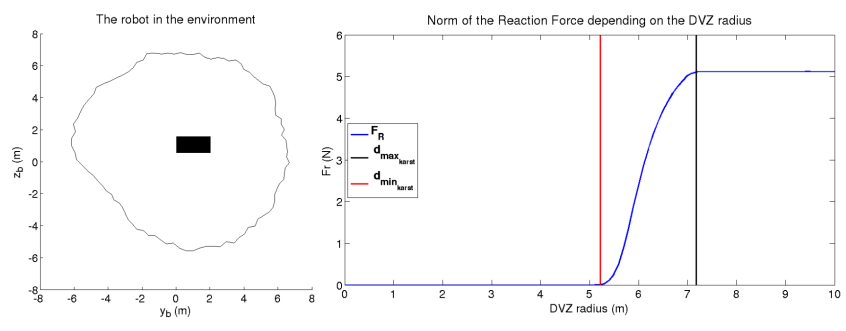

Fig. 4. Relationship between $\left\|\overrightarrow{F_{R}}\right\|$ and the DVZ radius

part of the figure. So if the robot is at a distance $d_{\text {max }}$ karst of the center, the force no longer depends of $R_{D V Z}$ when :

$$
R_{D V Z}>2 * d_{\text {max }_{\text {karst }}}
$$

Thus due to Assumption 5 we have :

$$
\begin{gathered}
R_{D V Z}=d_{\text {max }_{\text {capt }}} \\
\text { IV. CONTROL }
\end{gathered}
$$

\section{A. Intrusion and resulting force computation}

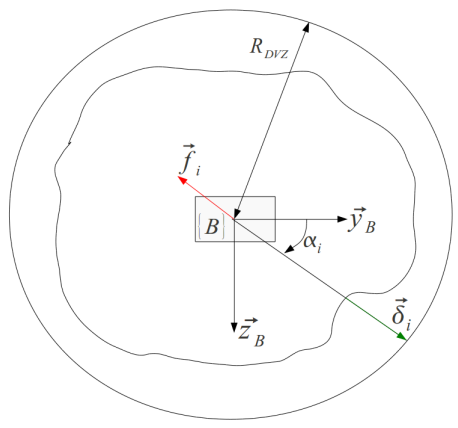

Fig. 5. Intrusion and force.

Let the set of intrusions in the DVZ be :

$I=\left\{\left(\overrightarrow{\delta_{i}}, \alpha_{i}\right)\right.$ such that $\left.\left\|\overrightarrow{\delta_{i}}\right\|=\delta_{i}=R_{D V Z}-d_{i}, i=1 . . N_{R}\right\}$

Each intrusion induces a reaction force, $\vec{f}_{i}$, such that :

$$
\overrightarrow{f_{i}}=-K_{D V Z} \overrightarrow{\delta_{i}}
$$

Where $K_{D V Z}$ is a strictly positive spring stiffness which shall be set up by the designer to get the desired system reponse profile.

The coordinates of this vector in $\{B\}$ are :

$$
\begin{aligned}
& f_{i_{y}}=-K_{D V Z} \cos \left(\alpha_{i}\right) \delta_{i} \\
& f_{i_{z}}=-K_{D V Z} \sin \left(\alpha_{i}\right) \delta_{i}
\end{aligned}
$$

The force resulting from all the intrusions is given by :

$$
\overrightarrow{F_{R}}=\sum_{i=1}^{N_{R}} \vec{f}_{i}
$$

Its coordinates in $\{B\}$ are :

$$
\begin{aligned}
& F_{R_{y}}=-K_{D V Z} \sum_{i=1}^{N_{R}} \cos \left(\alpha_{i}\right) \delta_{i} \\
& F_{R_{z}}=-K_{D V Z} \sum_{i=1}^{N_{R}} \sin \left(\alpha_{i}\right) \delta_{i}
\end{aligned}
$$




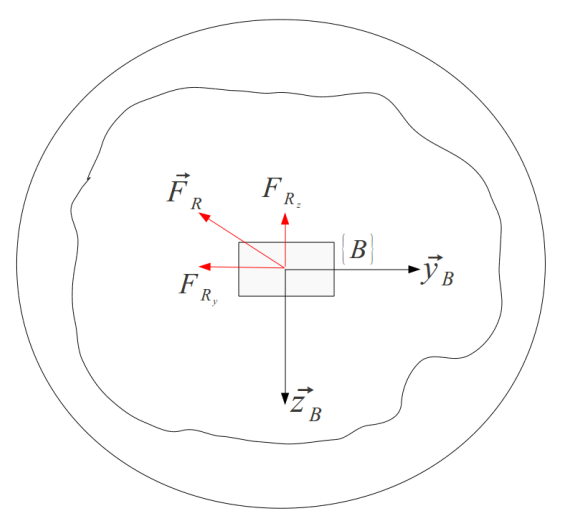

Fig. 6. Resulting force

\section{B. Control computation}

The resulting force $\overrightarrow{F_{R}}$ is not null due to the imbalance between the intrusions. Our aim is to compute the control in order to reach the point where :

$$
\overrightarrow{F_{R}}=\overrightarrow{0}
$$

As $\{B\}$ is an orthonormal frame, this objective becomes :

$$
\begin{aligned}
& F_{R_{y}}=0 \\
& F_{R_{z}}=0
\end{aligned}
$$

Moreover, we want our system to react to those forces like the mass-spring-damper system presented in section III alongside both $\overrightarrow{y_{B}}$ and $\overrightarrow{z_{B}}$. We can then write the two first control equations :

$$
\begin{gathered}
\dot{v}_{c o}=\frac{F_{R_{y}}}{m_{v}}-\frac{c_{v} v}{m_{v}} \\
\dot{w}_{c o}=\frac{F_{R_{z}}}{m_{w}}-\frac{c_{w} w}{m_{w}}
\end{gathered}
$$

where $m_{v}$ and $m_{w}$ are the robot's total masses (i.e. dry mass plus added mass) on $\overrightarrow{y_{B}}$ and $\overrightarrow{z_{B}}$ respectively. $c_{v}$ and $c_{w}$ are the damping coefficients.

As presented in section II, in addition to reaching the karst "center", we want our robot to reach a desired $\phi^{d}$. The evolution of the error $\phi^{d}-\phi$ is guided by the first-order differential equation :

$$
\left(\dot{\phi}^{d}-\dot{\phi}\right)=-K_{\phi}\left(\phi^{d}-\phi\right)
$$

where $K_{\phi}$ is a strictly positive parameter which drives the system response time.

As we set $\phi^{d}$ as a constant, we obtain from (4) :

$$
p^{d}=-K_{\phi}\left(\phi-\phi^{d}\right)
$$

Then similarly to (25), we can write :

$$
\left(\dot{p^{d}}-\dot{p}\right)=-K_{p}\left(p^{d}-p\right)
$$

where $K_{p}$ is a strictly positive parameter which drives the system response time.

Thus we obtain the last control equation :

$$
\dot{p}_{c o}=-K_{p} p-K_{r}\left(\phi-\phi^{d}\right)
$$

with $K_{r}=K_{p} K_{\phi}$ a strictly positive parameter.

Then we feed the desired accelerations $\dot{v}_{c o}, \dot{w}_{c o}$ and $\dot{p}_{c o}$ in the inverse dynamic model presented in (5), (6) and (7) to compute the forces that should be applied by the robot actuators (with Assumption 3 respected) :

$$
\begin{gathered}
F_{v_{c o}}=m_{v} \dot{v}_{c o}-d_{v} v=F_{R_{y}}-\left(c_{v}+d_{v}\right) v \\
F_{w_{c o}}=m_{w} \dot{w}_{c o}-d_{w} w=F_{R_{z}}-\left(c_{w}+d_{w}\right) w \\
\Gamma_{p_{c o}}=m_{p} \dot{p}_{c o}-d_{p} p=-\left(m_{p} K_{p}+d_{p}\right) p-K_{r} m_{p}\left(\phi-\phi^{d}\right)
\end{gathered}
$$

\section{Damping coefficient setting}

We shall now set up our damping coefficients, $c_{v}$ and $c_{w}$, to guaranty that our mass-spring-damper will work in a critical regime in the worst intrusion case. This will guaranty us that no matter how strong the intrusion may be, our system will converge without oscillation or overshooting.

Assuming that $N_{R}$ is even and thanks to the DVZ symmetric shape (18) becomes :

$$
F_{R_{y}}=-K_{D V Z} \sum_{i=1}^{\frac{N_{R}}{2}} \cos \left(\alpha_{i}\right)\left[\delta_{i}-\delta_{i+\frac{N_{R}}{2}}\right]
$$

Let us note :

$$
\Delta=\max _{i=1 \ldots \frac{N_{R}}{2}}\left[\delta_{i}-\delta_{i+\frac{N_{R}}{2}}\right]
$$

The worst intrusion case occurs when :

$$
\left[\delta_{i}-\delta_{i+\frac{N_{R}}{2}}\right]=\Delta \text { for all } i \in\left[1 . . \frac{N_{R}}{2}\right]
$$

Consequently we can write :

$$
\begin{array}{r}
F_{R_{y}} \leq F_{R_{y_{M a x}}} \\
\text { with } F_{R_{y_{M a x}}}=-K_{D V Z} \Delta \sum_{i=1}^{\frac{N_{R}}{2}} \cos \left(\alpha_{i}\right)
\end{array}
$$

And as $\cos \left(\alpha_{i}\right) \leq 1$ we can write the force in the worst case of intrusion :

$$
F_{R_{y_{M a x}}}<-K_{D V Z} \Delta \frac{N_{R}}{2}
$$

This leads to the computation of $c_{v}$ in order to guarantee a convergence without oscillation :

$$
c_{v}=2 \sqrt{m_{v} K_{D V Z} \frac{N_{R}}{2}}
$$

Similarly we can write :

$$
c_{w}=2 \sqrt{m_{w} K_{D V Z} \frac{N_{R}}{2}}
$$

Thus our resulting force is assimilated to the force a single spring of stiffness $K_{e}$ would generate. In the worst case $\left(K_{e}=K_{D V Z} \frac{N_{R}}{2}\right)$, the damping coefficients warrant convergence in critical regime. And in most cases $\left(K_{e}<\right.$ $K_{D V Z} \frac{N_{R}}{2}$ ), the generated force will be inferior to the maximal force. The system will be over-damped thus preventing any possibility of oscillations or overshooting. 


\section{Proof of stability}

Taking back the notations of [9], we can write the state of our controller as $(\pi, \Xi)$ with $\pi=(p, v, w, \phi)$ the states related to the robot dynamics and $\Xi=\left(F_{R_{y}}, F_{R_{z}}\right)$ the states related to the interaction of the DVZ with the environment.

We shall now prove the stability of our controller, which is an autonomous system. Consider the candidate Lyapunov function, we have to prove that it respects the conditions of Barbalat's lemma :

$$
\begin{array}{r}
V=\frac{\left(p-p^{d}\right)^{2}}{2}+2 K_{r} \frac{\left(\phi-\phi^{d}\right)^{2}}{2}+\frac{F_{R_{y}}^{2}}{2}+K_{e} m_{v} \frac{v^{2}}{2} \\
+\frac{F_{R_{z}}^{2}}{2}+K_{e} m_{w} \frac{w^{2}}{2}>0
\end{array}
$$

Its derivative is :

$$
\begin{aligned}
\dot{V} & =\left(\dot{p}-\dot{p}^{d}\right)\left(p-p^{d}\right)+2 K_{r}\left(\phi-\phi^{d}\right)\left(\dot{\phi}-\dot{\phi}^{d}\right) \\
& +F_{R_{y}} \dot{F}_{R_{y}}+K_{e} m_{v} \dot{v} v+F_{R_{z}} \dot{F}_{R_{z}}+K_{e} m_{w} \dot{w} w
\end{aligned}
$$

We have :

$$
\begin{array}{r}
\dot{F}_{R_{y}}=-K_{e} v \\
\dot{F}_{R_{z}}=-K_{e} w \\
K_{e}=\eta K_{D V Z} \text { such that } 0<\eta<\frac{N_{R}}{2}
\end{array}
$$

Remembering that $\dot{\phi}^{d}=0$ and with (27) verified, we can write from (26), (23), (24) and (4) :

$$
\dot{V}=-K_{p} p^{2}-K_{p} K_{\phi}^{2}\left(\phi-\phi^{d}\right)^{2}-K_{e} c_{v} v^{2}-K_{e} c_{w} w^{2}
$$

Thus $\dot{V}<0$. We have proven that $V$ is bounded. Derivating $\dot{V}$, we can write :

$$
\begin{aligned}
\ddot{V}=-2 K_{p} \dot{p} p-2 K_{p} K_{\phi}^{2}( & \left.\phi-\phi^{d}\right)\left(\dot{\phi}-\dot{\phi}^{d}\right) \\
& -2 K_{e} c_{v} v \dot{v}-2 K_{e} c_{w} w \dot{w}
\end{aligned}
$$

From (28), (4), (23) and (24), $\ddot{V}$ becomes :

$$
\begin{gathered}
\ddot{V}=2 K_{p}^{2} p^{2}+\left(\phi-\phi^{d}\right) p\left(2 K_{p} K_{r}-2 K_{r} K_{\phi}\right)+2 \frac{K_{e} c_{v}^{2}}{m_{v}} v^{2} \\
-2 \frac{K_{e} c_{v}}{m_{v}} v F_{R_{y}}+2 \frac{K_{e} c_{w}^{2}}{m_{w}} w^{2}-2 \frac{K_{e} c_{w}}{m_{w}} w F_{R_{z}}
\end{gathered}
$$

As $V$ is bounded this means $p,\left(\phi-\phi^{d}\right), v, w, F_{R_{y}}$ and $F_{R_{z}}$ are bounded. Thus $\ddot{V}$ is bounded.

We have proven that $V>0, \dot{V}<0$ and $\ddot{V}$ is bounded. We can use Barbalat's lemma [11] and say that our control is Globally Uniformly Assimptotically Stable.

\section{Simulation RESUlts}

\section{A. Simulation parameters}

We are here listing the value of the differents parameters shared by the two simulations presented below.

The robot moves with $u=0.5 \mathrm{~m} / \mathrm{s}$. We are using a profilometry sonar whose parameters are : $T_{c}=1 \mathrm{~s}$, $d_{\text {max }_{\text {capt }}}=15 \mathrm{~m}, d_{\text {min }_{\text {capt }}}=0.1 \mathrm{~m}$ and $N_{R}=100$. The

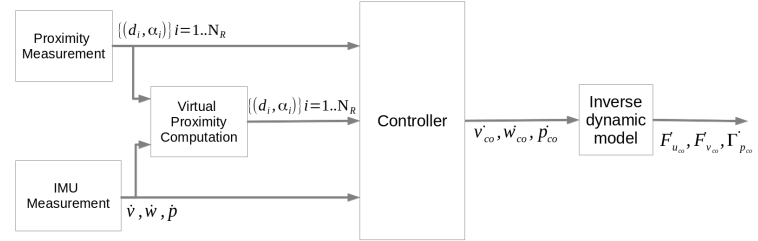

Fig. 7. Controller and sensors synoptic. The computation of $v, w, p$ and $\phi$ by integration of the measured accelerations is made in the controller.

robot speeds and angle are computed using an IMU of sampling time $T_{I M U}=0.1 \mathrm{~s}$.

Our control loop has a period of $0.1 \mathrm{~s}$. This means that in between two sonar measurements, we must create a virtual measurement from the latest proximity measurement and the current estimated robot position (obtained from integration of the IMU measurements) as shown in Figure 7.

For both simulations, We have set $m_{v}=m_{w}=15 \mathrm{~kg}$, $m_{p}=1 \mathrm{~kg}$ and $d_{v}=d_{w}=d_{p}=-1$. The three designer defined parameters, $K_{D V Z}, K_{r}$ and $K_{p}$ have the value : $K_{D V Z}=0.8, K_{r}=2$ and $K_{p}=4$. Finally, $\phi^{d}=0$.

\section{B. Pipe simulation}

Our first simulation example is a regular pipe made of four cylinders as shown in Figure 8. This environment will allow us to demonstrate the convergence properties of our system.

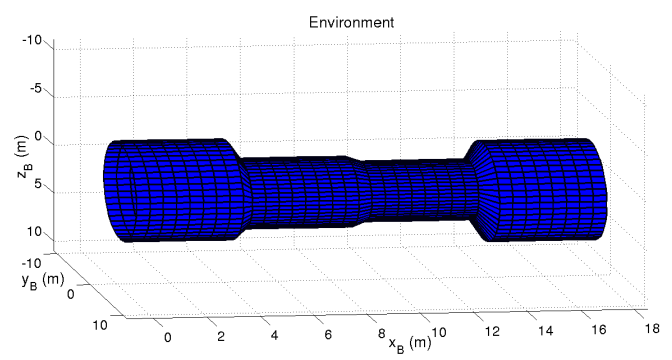

Fig. 8. First Simulation Environment : A pipe made of four cylinders of different radii and center coordinates.

The Figure 9 presents the evolution of the robot pose. In the two upper figures, as expected from the pipe regular shape, the robot converges to the center of the pipe. Moreover, as shown by the lower right figure, $F_{R_{y}}$ and $F_{R_{z}}$ converges to 0 as expected. Finally the lower left picture illustrates the convergence of $\phi$ towards $\phi^{d}=0$.
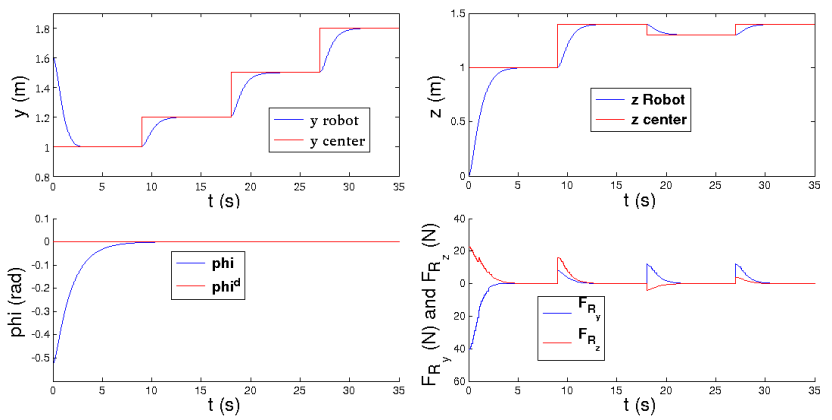

Fig. 9. Pose, $F_{R_{y}}, F_{R_{z}}$ evolution in the pipe environment simulation 
The Figure 10 presents the forces generated by the control activity. They have values compatible with the forces our real system (the Jack, (C)Ciscrea) could generate.

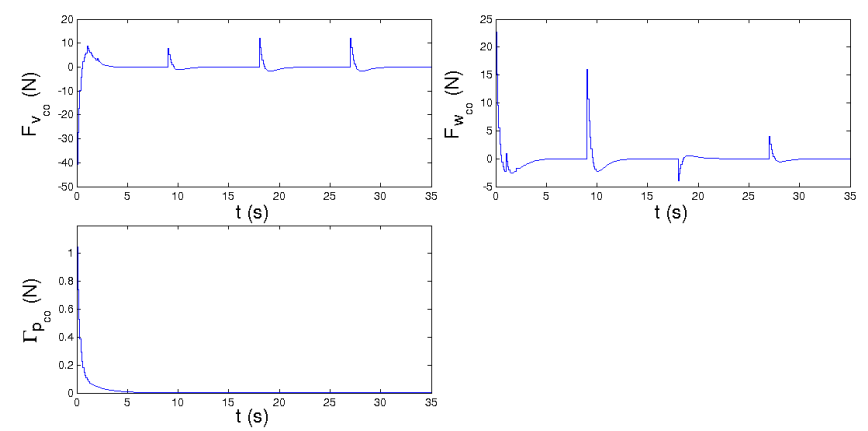

Fig. 10. Actuation activity in the pipe environment simulation

\section{Karst environment simulation}

The second simulation uses the karstic environment presented in Figure 11.

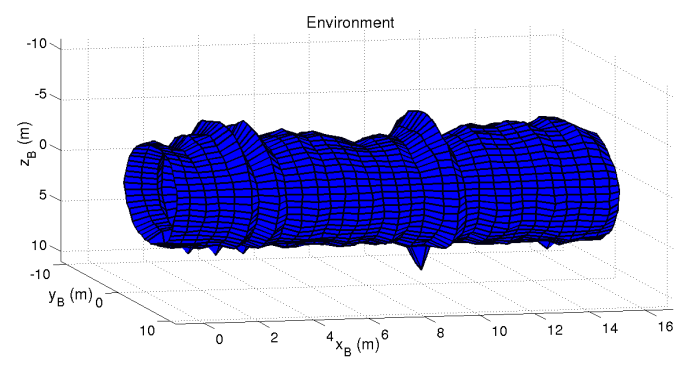

Fig. 11. Karstic environment

In the two upper figures of Figure 12, we see that our system tracks the measurement "center" but cannot converge because the environement is evolving too quickly. And as one can see on the lower right figure, $F_{R_{y}}$ and $F_{R_{z}}$ also don't reach 0 but they clearly evolve towards 0 after the environment changes.
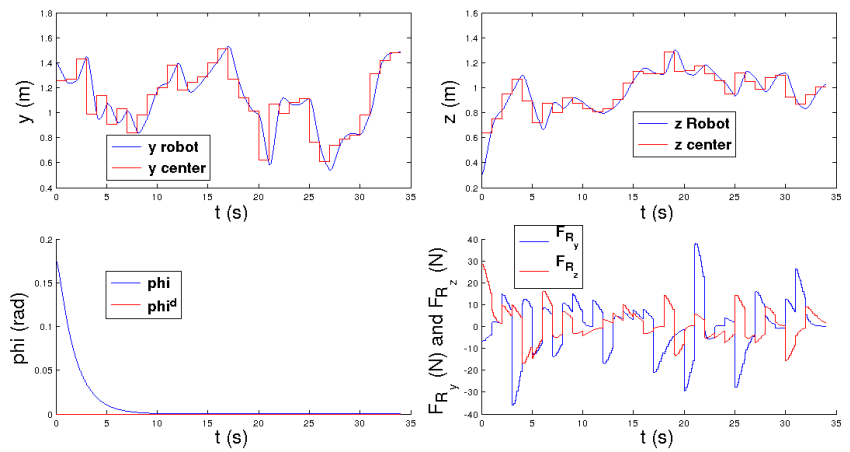

Fig. 12. Pose, $F_{R_{y}}, F_{R_{z}}$ evolution in the Karstic environment simulation

The Figure 13 presents the forces generated by the robot's actuation system which are still realistic.
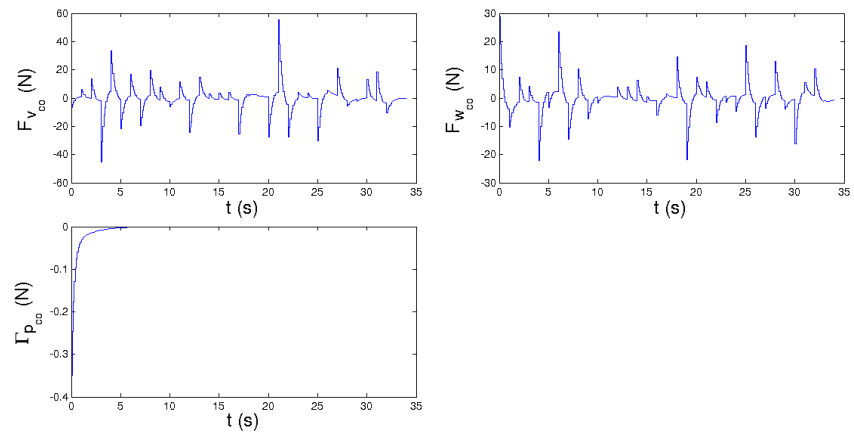

Fig. 13. Actuation activity in the Karstic environment simulation

\section{CONCLUSION AND FUTURE WORK}

In this paper, we have presented a new control algorithm allowing an AUV to center reactively in a conduit of a karstic aquifer. It is based on the DVZ principle and allows our dynamic system to behave like a mass-spring-damper system. Moreover, it requires the user to set up only three control parameters. We have proven the stability of such a controller. Simulations have demonstrated the good convergence and dynamic abilities of our controller in regularly or irregularly shaped environments. We should now study the robustness of our controller to modelling uncertainties, sensor noise and environmental disturbances. We should also study the interaction between this control algorithm and the one presented in Assumption 1. Finally we will try to relax Assumption 5.

\section{ACKNOWLEDGEMENTS}

The authors would like to thank the French labex NUMEV for supporting this research.

\section{REFERENCES}

[1] N. Dorfliger, H. Jourde, B. Ladouche, P. Fleurry, P. Lachassagne, Y. Conroux, S. Pistre, and A.Vestier, "Active water management resources of karstic water catchment: the example of le lez spring," in World Water Congress, 2008.

[2] M. Bakalowicz, "Karst groudwater : a challenge for new ressources," Journal of Hydrogeology, pp. 13: 148-160, 2005.

[3] O. Tatar and D. Mandru, "Robotic systems for inspection and exploration," Annals of the Oradea University. Fascicule of Management and Technological Engineering, vol. VII (XVII), 2008.

[4] R. Bradbeer, S. Harrold, F. Nickols, and L. Yeung, "An underwater robot for pipe inspection," in Proceedings of 4th International Conference on Mechatronics and Machine Vision in Practice, (M2VIP97), 1997, pp. 152-156.

[5] T. Saitho, N. Tada, and R. Konishi, "Indoor mobile robot navigation by center following based on monocular vision," in Computer Vision, X. Zhihui, Ed. InTech, 2008, ch. 20, pp. 351-366.

[6] R. Carelli and E. O. Freire, "Corridor navigation and wall-following stable control for sonar-based mobile robots," Robotics and Autonomous Systems, no. 45, 2003.

[7] A. K. Simpson, "Autonomous robot control for underwater tunnel mapping," Senior thesis, Princetown University, 2012.

[8] L. Yingxue, "Autonomous control for remotely operated vehicles," Senior thesis, Princetown University, 2012.

[9] R. Zapata and P. Lepinay, "Fast mobile robots in unstructured environments," Lecture Notes in Computer Science, vol. 708, pp. 94-105, 1993.

[10] R. Carelli, H. Secchi, and V. Mut, "Algorithms for stable control of mobile robots with obstacle avoidance," Latin America Applied Research, vol. 29, pp. 191-196, 1999.

[11] J. Slotine and W. Li, Applied Nonlinear Control. Prentice-Hall, 1991. 